$\xi=\square$

\title{
Solutions of Kudryashov - Sinelshchikov equation and generalized Radhakrishnan-Kundu-Lakshmanan equation by the first integral method
}

\author{
S. Subhaschandra Singh * \\ Department of Physics, Imphal College, Imphal, Manipur, India \\ *Corresponding author E-mail: subhasic@yahoo.co.in
}

\begin{abstract}
This paper shows the applicability of the First Integral Method in obtaining solutions of Nonlinear Partial Differential Equations (NLPDEs). The method is applied in constructing solutions of Kudryashov-Sinelshchikov equation (KSE) and Generalized Radhakrishnan-Kundu-Lakshmanan Equation (GRKLE). The First Integral Method, which is based on the Ring Theory of Commutative Algebra, is a direct algebraic method for obtaining exact solutions of NLPDEs. This method is applicable to integrable as well as nonintegrable NLPDEs. The method is an efficient method for obtaining exact solutions of many Nonlinear Evolution Equations (NLEEs).
\end{abstract}

Keywords: Division Theorem; First Integral Method; Generalized Radhakrishnan - Kundu- Lakshmanan Equation (GRKLE); Kudryashov - Sinelshchikov Equation (KSE); NLEEs; Optical Solitons.

\section{Introduction}

Nonlinear Evolution Equations (NLEEs) are frequently encountered in the study of many complex phenomena in various branches of physics such as Biophysics, Condensed Matter Physics, Fluid Physics, Neurophysics, Nonlinear Optics, Particle Physics, Plasma Physics, Quantum Field Theory, etc. and in many other branches of science such as Ecology, Physiology etc. as well as in Economics and Social Science. In the recent a few decades, quite a number of methods had been suggested so far for finding exact solutions of NLEEs in Mathematical Physics and the First Integral Method is one of them. The first integral method, which is based on the ring theory of commutative algebra, was first proposed by Z.S. Feng [1] and was further developed by himself [2 - 4]. This method has been applied by many authors in solving different types of NLEEs encountered in science and engineering [5 - 18]. In the present paper, the first integral method is applied in solving the Kudryashov-Sinelshchikov Equation (KSE) and the Generalized Radhakrishnan- Kundu-Lakshmanan Equation (GRKLE). The rest of the article is arranged as in the following. In section 2, a brief description of the method is presented. In section 3, the method is applied in obtaining the solutions of KSE [19 - 22] and GRKLE [23-25]. In section 4, a brief conclusion is presented.

\section{The first integral method}

Let us consider a general NLPDE in the form

$F\left(u, u_{x}, u_{t}, u_{x x}, u_{x t}, u_{t t}, u_{x x x}, \ldots\right)=0$,

where $u=u(x, t)$ is its solution, $x$ and $t$ represent the spatial and the temporal variables.

Let us introduce the transformations, $u=u(x, t)=U(\xi), \xi=x-v t$,

where $v$ is a constant to be determined latter.

Now, we have,

$$
\begin{aligned}
& \frac{\partial}{\partial x}(.)=\frac{d}{d \xi}(.), \frac{\partial}{\partial t}(.)=-v \frac{d}{d \xi}(.) \\
& \frac{\partial^{2}}{\partial x^{2}}(.)=\frac{d^{2}}{d \xi^{2}}(.), \frac{\partial^{2}}{\partial x \partial t}(.)=-v \frac{d^{2}}{d \xi^{2}}(.), \text { etc. }
\end{aligned}
$$

Using equations (3), we transform the NLEE (1) into a nonlinear ordinary differential equation (NLODE) of the form,

$G\left(U, U^{\prime}, U^{\prime \prime}, U^{\prime \prime \prime}, \ldots\right)=0$

where the primes denote derivatives with respect to the same variable $(\xi)$ such that

$U^{\prime}=\frac{d U}{d \xi}, U^{\prime \prime}=\frac{d^{2} U}{d \xi^{2}}$, etc.

Let us suppose that the solution of the Non Linear Ordinary Differential Equation (NLODE) (4) can be expressed as

$u(x, t)=U(\xi)=f(\xi)$.

We further introduce the following new independent variables

$X(\xi)=f(\xi), Y(\xi)=f^{\prime}(\xi)=\frac{d f}{d \xi}=\frac{d X}{d \xi^{\prime}}$

leading to a system,

$X^{\prime}(\xi)=Y(\xi), \quad Y^{\prime}(\xi)=H(X(\xi), Y(\xi))$. 
If we can find two first integrals to the system of equations (7) under the same conditions, then the analytic solutions of equations (7) can be obtained directly. However, in general, it is really difficult for us to realize this even for one first integral, because for a given plane autonomous system, there exists neither a systematic theory that can tell us how to find its first integrals nor a logical way for telling us what these first integrals are. We will apply the Division Theorem to obtain a first integral to the system of equations (7) which reduces eqn. (4) to a first order integrable ODE. An exact solution of eqn. (1) is then obtained by solving this ODE. For convenience, let us recall the division theorem for two variables in the complex domain $C[w, z]$.

Division Theorem: For two polynomials $P(w, z)$ and $Q(w, z)$ in a complex domain $C[w, z]$, if $P(w, z)$ is irreducible in $C[w, z]$ and if $Q(w, z)$ vanishes at all zero points of $P(w, z)$, then there exists another polynomial $G(w, z)$ in $C[w, z]$ such that $Q(w, z)=P(w, z)$ $G(w, z)$. The division theorem follows immediately from Hilbert Nullstellensatz theorem of commutative algebra.

\section{Applications of the first integral method}

The first integral method is applicable in solving various NLPDEs and systems of NLPDEs. Using this method, solutions of Kudryashov - Sinelshchikov Equation (KSE) and Generalized Radhakrishnan - Kundu - Lakshmanan Equation (GRKLE) are obtained as in the following.

\subsection{Kudryashov- Sinelshchikov Equation (KSE)}

The KSE [19 - 22] describes pressure waves in a liquid with gas bubbles taking into consideration the viscosity of the liquid and the heat transfer. The equation reads

$$
u_{t}+\alpha u u_{x}+u_{x x x}-\beta\left(u u_{x x}\right)_{x}-\gamma u_{x} u_{x x}=0,
$$

where $\alpha, \beta, \gamma$ are real parameters.

Using the transformation $u(x, t)=U(\xi)$ with $\xi=x-v$ t and also the relations (3), eqn. (8) yields

$$
-v U^{\prime}+\alpha U U^{\prime}+U^{\prime \prime \prime}-\beta\left(U U^{\prime \prime}\right)^{\prime}-\gamma U^{\prime} U^{\prime \prime}=0,
$$

where the primes denote differentiation with respect to $\xi$. Integrating both sides of eqn. (9) once with respect to $\xi$ and choosing the integration constant as zero, we obtain

$-v U+\frac{\alpha}{2} U^{2}+U^{\prime \prime}-\beta U U^{\prime \prime}-\frac{\gamma}{2} U^{\prime 2}=0$.

Using eqns. (6) and (7), we obtain

$$
\begin{aligned}
& X^{\prime}(\xi)=\frac{d X(\xi)}{d \xi}=Y(\xi), \\
& Y^{\prime}(\xi)=\frac{d Y(\xi)}{d \xi} \\
& =\frac{1}{1-\beta X(\xi)}\left[\begin{array}{c}
v X(\xi)-\frac{\alpha}{2} X^{2}(\xi) \\
+\frac{\gamma}{2} Y^{2}(\xi)
\end{array}\right] .
\end{aligned}
$$

Now, let us introduce another transformation,

$d \xi=(1-\beta X) d \eta$ where $\eta$ is a new variable.

Then, eqn. (11) yields

$$
\frac{d X}{d \eta}=(1-\beta X) Y, \frac{d Y}{d \eta}=v X-\frac{\alpha}{2} X^{2}+\frac{\gamma}{2} Y^{2} .
$$

To seek the first integral of the system of eqns. (13), we are to apply the division theorem. Let us suppose that $X=X(\eta)$ and $Y=$
$Y(\eta)$ are nontrivial solutions of eqn. (13), and $q(X, Y)=$ $\sum_{j=0}^{m} a_{j}(X) Y^{j}$ is an irreducible polynomial in $C[X, Y]$ such that

$q(X(\eta), Y(\eta))=\sum_{j=0}^{m} a_{j}(X(\eta)) Y^{j}(\eta)=0$,

where $a_{j}(X)(j=0,1,2,3, \ldots m)$ are polynomials in $X$ with $a_{m}(X) \neq 0$. Here, eqn. (14) is called the first integral of eqn. (13). It is noted that $\frac{d q}{d \eta}$ is a polynomial in $X$ and $Y$ and that $\frac{d q}{d \eta}=0$. Now according to the division theorem, there exists a polynomial $g(X)+h(X) Y$ in $C[X, Y]$ such that from eqns.(13) and (14), we have,

$\frac{d q}{d \eta}=\frac{d q}{d X} \frac{d X}{d \eta}+\frac{d q}{d Y} \frac{d Y}{d \eta}$

$=\left\{\sum_{j=0}^{m} a_{j}^{\prime}(X) Y^{j}\right\}\{(1-\beta X) Y\}$

$+\left\{\sum_{j=0}^{m} j a_{j}(X) Y^{j-1}\right\}\left\{v X-\frac{\alpha}{2} X^{2}+\frac{\gamma}{2} Y^{2}\right\}$

$=\{g(X)+h(X) Y\} \sum_{j=0}^{m} a_{j}(X) Y^{j}$,

where the prime denotes differentiation with respect to $X$.

For $m=1$, we have,

$$
\begin{aligned}
& a_{0}^{\prime}(X)(1-\beta X) Y+a_{1}^{\prime}(X)(1-\beta X) Y^{2} \\
& +a_{1}(X)\left\{v X-\frac{\alpha}{2} X^{2}+\frac{\gamma}{2} Y^{2}\right\} \\
& =\{g(X)+h(X) Y\}\left\{a_{0}(X)+a_{1}(X) Y\right\} .
\end{aligned}
$$

Equating coefficients of $Y^{j}(j=2,1,0)$ from both sides of equation (16), we obtain,

$$
\begin{aligned}
& (1-\beta X) a_{1}^{\prime}(X)=\left\{h(X)-\frac{\gamma}{2}\right\} a_{1}(X), \\
& (1-\beta X) a_{0}^{\prime}(X)=g(X) a_{1}(X)+h(X) a_{0}(X), \\
& g(X) a_{0}(X)=a_{1}(X)\left(v X-\frac{\alpha}{2} X^{2}\right),
\end{aligned}
$$

where the primes denote differentiation with respect to $X$.

Since $a_{j}(X)(j=0,1)$ are polynomials, we deduce from eqn. (17a) that $h(X)=\gamma / 2$ and $a_{1}(X)=$ constant. For simplicity, we take $a_{1}(X)=1$. Balancing the degrees of $g(X)$ and $a_{0}(X)$ in eqn. (17b), we conclude that $\operatorname{deg}(g(X)) \leq \operatorname{deg}\left(a_{0}(X)\right)=1$. Let us assume that

$$
\begin{aligned}
& g(X)=A_{0}+A_{1} X\left(A_{1} \neq 0\right), \\
& a_{0}(X)=B_{0}+B_{1} X\left(B_{1} \neq 0\right),
\end{aligned}
$$

Now, substituting the values of $a_{0}(X), a_{0}^{\prime}(X), a_{1}(X), g(X)$ and $h(X)$ into eqn. (17b), we obtain,

$B_{1}-\beta B_{1} X=A_{0}+A_{1} X+\frac{\gamma}{2} B_{0}+\frac{\gamma}{2} B_{1} X$.

Equating coefficients of $X^{j}(j=0,1)$ from both sides, we obtain

$A_{0}=B_{1}-\frac{\gamma}{2} B_{0}$

$A_{1}=-\left(\beta+\frac{\gamma}{2}\right) B_{1}$.

Substituting the values of $A_{0}$ and $A_{1}$ into eqn. (18), we obtain,

$$
g(X)=B_{1}-\frac{\gamma}{2} B_{0}-\left(\beta+\frac{\gamma}{2}\right) B_{1} X .
$$


Substituting this value of $g(X)$ and also the values of $a_{0}(X)$ and $a_{1}(X)$ into eqn. (17c), we obtain,

$\left\{B_{1}-\frac{\gamma}{2} B_{0}-\left(\beta+\frac{\gamma}{2}\right) B_{1} X\right\}\left\{B_{0}+B_{1} X\right\}$

$=v X-\frac{\alpha}{2} X^{2}$,

Equating coefficients of $X^{j}(j=0,1,2)$ to zero, we obtain the following set of nonlinear algebraic equations,

$B_{0} B_{1}-\frac{\gamma}{2} B_{0}^{2}=0$,

$B_{1}^{2}-(\beta+\gamma) B_{0} B_{1}-v=0$,

$\frac{\alpha}{2}-\left(\beta+\frac{\gamma}{2}\right) B_{1}^{2}=0$.

The solutions of the set of eqns. (24) are

$B_{0}= \pm \frac{2}{\gamma} \sqrt{\frac{\alpha}{2 \beta+\gamma}}$

$B_{1}= \pm \sqrt{\frac{\alpha}{2 \beta+\gamma}}$

$v=-\frac{\alpha}{\gamma}$

Now eqn (14) yields,

$a_{0}+a_{1} Y=0$

Using eqns. (19), (25a) and (25b), eqn. (26) yields

$\pm \frac{2}{\gamma} \sqrt{\frac{\alpha}{2 \beta+\gamma}} \pm \sqrt{\frac{\alpha}{2 \beta+\gamma}} X+Y=0$.

As $Y=\frac{d X}{d \xi}$, we write,

$\frac{d X}{d \xi} \pm \sqrt{\frac{\alpha}{2 \beta+\gamma}} X=\mp \frac{2}{\gamma} \sqrt{\frac{\alpha}{2 \beta+\gamma}}$.

Here, Integrating Factor $=\exp \left( \pm \sqrt{\frac{\alpha}{2 \beta+\gamma}} \xi\right)$.

Eqn. (28) has the solution,

$X(\xi)=\mp \frac{2}{\gamma}+C \exp \left(\mp \sqrt{\frac{\alpha}{2 \beta+\gamma}} \xi\right)$.

Using eqns. (5), (6) and (25c), eqn. (29) yields,

$u(x, t)=\mp \frac{2}{\gamma}+C \exp \left[\mp\left(\sqrt{\frac{\alpha}{2 \beta+\gamma}}\right)\left(x+\frac{\alpha}{\gamma} t\right)\right]$.

Let us put $C=-\sqrt{\frac{2 \beta+\gamma}{\alpha}} \exp \left[\mp\left(\sqrt{\frac{\alpha}{2 \beta+\gamma}}\right) x_{0}\right]$

Then, we have,

$u(x, t)=\mp \frac{2}{\gamma}$

$-\sqrt{\frac{2 \beta+\gamma}{\alpha}} \exp \left[\mp\left(\sqrt{\frac{\alpha}{2 \beta+\gamma}}\right)\left(x_{0}+x+\frac{\alpha}{\gamma} t\right)\right]$.

Eqn. (31) is an exact solution of the Kudryashov - Sinelshchikov equation (8).

In a similar manner, we can obtain solutions for other values of $m$.

3.2. Generalized Radhakrishnan- Kundu- Lakshmanan equation (GRKLE)
The GRKLE [20] is generally encountered in the study of optical solitons in Nonlinear Fiber Optics. The equation reads

$i q_{t}+a q_{x x}+b|q|^{2 n} q-i\left[\lambda\left(|q|^{2 n} q\right)_{x}\right.$

$\left.+v\left(|q|^{2 n}\right)_{x} q-\gamma q_{x x x}\right]=0$.

Here, the parameter $n$ (positive integer) dictates the power law nonlinearity. If $n$ is unity, the power law nonlinearity reduces to the Kerr law nonlinearity. A special case of equation (32) with $n=$ 1 and $v=0$ is called the Radhakrishnan- Kundu- Lashmanan Equation (RKLE). In eqn. (32), $a, b, \lambda, v$, and $\gamma$ are all real constants. The first term represents the evolution term, the second and the third terms respectively represent the group velocity dispersion (GVD) and nonlinear terms, the fourth term represents the selfsteepening term, the fifth term represents the nonlinear dispersion term and the last term represents the third order dispersion term.

Let us assume the solution of eqn. (32) as

$q(x, t)=f(\xi) \exp [i\{\chi(\xi)-\omega t\}]$,

where $\chi(\xi)=\beta \xi+x_{0}, \xi=x-v t$

and $f(\xi)$ is a function to be determined latter.

Now, we have,

$i q_{t}=[(\beta v+\omega) f(\xi)$

$\left.-i v f^{\prime}(\xi)\right] \exp [i\{\chi(\xi)-\omega t\}]$,

$a q_{x x}=\left[-a \beta^{2} f(\xi)+2 i a \beta f^{\prime}(\xi)\right.$

$\left.+a f^{\prime \prime}(\xi)\right] \exp [i\{\chi(\xi)-\omega t\}]$

$b|q|^{2 n} q=b f^{2 n+1}(\xi) \exp [i\{\chi(\xi)-\omega t\}]$,

$i \lambda\left(|q|^{2 n} q\right)_{x}=\left[\begin{array}{c}-\beta \lambda f^{2 n+1}(\xi) \\ +i(2 n+1) \lambda f^{2 n}(\xi) f^{\prime}(\xi)\end{array}\right] \exp [i\{\chi(\xi)-$

$\omega t\}]$,

$i v\left(|q|^{2 n}\right)_{x} q$

$=\left[2 i v n f^{2 n}(\xi) f^{\prime}(\xi)\right] \exp [i\{\chi(\xi)-\omega t\}]$,

$-i \gamma q_{x x x}=\left[\begin{array}{l}-\beta^{3} \gamma f(\xi)+3 \beta \gamma f^{\prime \prime}(\xi) \\ +3 i \beta^{2} \gamma f^{\prime}(\xi)-i \gamma f^{\prime \prime \prime}(\xi)\end{array}\right]$

$\times \exp [i\{\chi(\xi)-\omega t\}]$

Substituting eqns. (35) to (40) into eqn. (32), we obtain,

$(\beta v+\omega) f(\xi)-i v f^{\prime}(\xi)-a \beta^{2} f(\xi)+2 i a \beta f^{\prime}(\xi)+$ $a f^{\prime \prime}(\xi)+b f^{2 n+1}(\xi)$

$+\beta \lambda f^{2 n+1}(\xi)-i(2 n+1) \lambda f^{2 n}(\xi) f^{\prime}(\xi)-$

$2 i v n f^{2 n}(\xi) f^{\prime}(\xi)+\beta^{3} \gamma f(\xi)$

$-3 i \beta^{2} \gamma f^{\prime}(\xi)-3 \beta \gamma f^{\prime \prime}(\xi)+i \gamma f^{\prime \prime \prime}(\xi)=0$.

From eqn. (41), equating the real and the imaginary parts separately to zero, we obtain,

Real Part:

$(a-3 \beta \gamma) f^{\prime \prime}(\xi)+\left(\beta v+\omega-a \beta^{2}+\beta^{3} \gamma\right) f(\xi)$

$+(b+\beta \lambda) f^{2 n+1}(\xi)=0$.

Imaginary Part: 


$$
\begin{aligned}
& \gamma f^{\prime \prime \prime}(\xi)+\left(2 a \beta-v-3 \beta^{2} \gamma\right) f^{\prime}(\xi) \\
& -\{(2 n+1) \lambda+2 v n\} f^{2 n}(\xi) f^{\prime}(\xi)=0 .
\end{aligned}
$$

Integrating eqn. (43) once with respect to $\xi$ and choosing the integration constant as zero, we obtain,

$\gamma f^{\prime \prime}(\xi)+\left(2 a \beta-v-3 \beta^{2} \gamma\right) f(\xi)$

$-\frac{\{(2 n+1) \lambda+2 v n\}}{2 n+1} f^{2 n+1}(\xi)=0$.

As the same function $f(\xi)$ satisfies both the equations (42) and (44), we obtain the following constraint conditions

$$
\frac{(a-3 \beta \gamma)}{\gamma}=\frac{\left(\beta v+\omega-a \beta^{2}+\beta^{3} \gamma\right)}{2 a \beta-v-3 \beta^{2} \gamma}=-\frac{(b+\beta \lambda)(2 n+1)}{(2 n+1) \lambda+2 v n} .
$$

Thus, instead of solving both the eqns. (42) and (44), we have to solve either of the two eqns. Let us solve eqn. (44) under the constraint conditions of eqns. (45).

Let us put $f(\xi)=U^{\frac{1}{n}}(\xi)$.

Then, we have,

$$
\begin{aligned}
& f^{\prime}(\xi)=\frac{1}{n} U^{\frac{1}{n}-1}(\xi) U^{\prime}, \\
& f^{\prime \prime}(\xi)=\frac{(1-n)}{n^{2}} U^{\frac{1-2 n}{n}} U^{\prime 2}+\frac{1}{n} U^{\frac{1-n}{n}} U^{\prime \prime},
\end{aligned}
$$

where the primes indicate differentiation with respect to $\xi$. Using eqns. (46) and (47) in eqn. (44), we obtain,

$$
\begin{aligned}
& U^{\prime \prime}+\frac{1-n}{n} \frac{U^{\prime 2}}{U}+\frac{n\left(2 a \beta-v-3 \beta^{2} \gamma\right)}{\gamma} U \\
& -\frac{n\{(2 n+1) \lambda+2 v n\}}{\gamma(2 n+1)} U^{3}=0 .
\end{aligned}
$$

If we put $X=U(\xi), Y=\frac{d U}{d \xi}=\frac{d X}{d \xi}=X^{\prime}$,

eqn. (48) is equivalent to a two dimensional autonomous system,

$X^{\prime}=Y$,

$Y^{\prime}=\left(1-\frac{1}{n}\right) \frac{Y^{2}}{X}+\frac{n\left(3 \beta^{2} \gamma+v-2 a \beta\right)}{\gamma} X$

$+\frac{n\{(2 n+1) \lambda+2 v n\}}{\gamma(2 n+1)} X^{3}$.

Making the transformation

$$
d \eta=\frac{d \xi}{X},
$$

The system (50) becomes

$\frac{d X}{d \eta}=X Y$

and $\frac{d Y}{d \eta}=\frac{n\left(3 \beta^{2} \gamma+v-2 a \beta\right)}{\gamma} X^{2}+\frac{n\{(2 n+1) \lambda+2 v n\}}{\gamma(2 n+1)} X^{4}$

$+\left(1-\frac{1}{n}\right) Y^{2}$

Let us apply the Division Theorem to seek the first integral to system (52). Let us assume that $X=X(\eta)$ and $Y=Y(\eta)$ are the nontrivial solutions to the system (52). Further, let us assume that

$$
Q(X, Y)=\sum_{j=0}^{j=m} a_{j}(X) Y^{j}=0
$$

is an irreducible polynomial in the complex domain $C[X, Y]$ such that

$$
\begin{aligned}
& Q(X(\eta), Y(\eta))=\sum_{j=0}^{m} a_{j}(X(\eta)) Y^{j}(\eta)=0 \\
& \quad \text { where } a_{j}(X)(j=0,1,2, \ldots m) \text { are polynomials of } X \\
& \text { and } a_{m}(X) \neq 0 .
\end{aligned}
$$

Eqn. (54) is called the first integral to the system (52).

According to the Division Theorem, there exists a polynomial $g(X)+h(X) Y$ in the complex domain $C[X, Y]$ such that

$\frac{d Q}{d \eta}=\frac{d Q}{d X} \frac{d X}{d \eta}+\frac{d Q}{d Y} \frac{d Y}{d \eta}=\{g(X)+h(X) Y\} \sum_{j=0}^{m} a_{j}(X) Y^{j}$,

Or, $\sum_{j=0}^{m} a_{j}^{\prime}(X) X Y^{j+1}$

$+\sum_{j=0}^{m} \frac{n\left(3 \beta^{2} \gamma+v-2 a \beta\right)}{\gamma} j a_{j}(X) X^{2} Y^{j-1}$

$+\sum_{j=0}^{m} \frac{n\{(2 n+1) \lambda+2 v n\}}{\gamma(2 n+1)} j a_{j}(X) X^{4} Y^{j-1}$

$+\sum_{j=0}^{m}\left(1-\frac{1}{n}\right) j a_{j}(X) Y^{j+1}$

$=\sum_{j=0}^{m}\{g(X)+h(X) Y\} a_{j}(X) Y^{j}$,

where the primes denote differentiation with respect to $X$.

Let us consider the case with $m=1$. Then, equating the coefficients of $Y^{j}(j=2,1,0)$ from both sides of eqn. (55), we obtain,

$X a_{1}^{\prime}(X)=h(X) a_{1}(X)-\left(1-\frac{1}{n}\right) a_{1}(X)$,

$X a_{0}^{\prime}(X)=g(X) a_{1}(X)+h(X) a_{0}(X)$,

$a_{1}(X)\left[\frac{n\left(3 \beta^{2} \gamma+v-2 a \beta\right)}{\gamma} X^{2}+\frac{n\{(2 n+1) \lambda+2 v n\})}{\gamma(2 n+1)} X^{4}\right]$

$=g(X) a_{0}(X)$

Since $a_{j}(j=0,1)$ are polynomials, we deduce from eqn. (56a) that $a_{1}(X)$ is a constant and $h(X)=\left(1-\frac{1}{n}\right)$. For simplicity, we take, $a_{1}(X)=1$. Balancing the degrees of $g(X)$ and $a_{0}(X)$, we conclude that $\operatorname{deg}[g(X)]=\operatorname{deg}\left[a_{0}(X)\right]=2$. Let us suppose that

$a_{0}(X)=A_{0}+A_{1} X+A_{2} X^{2}$

where $A_{0}, A_{1}, A_{2}\left(A_{2} \neq 0\right)$ are arbitrary constants to be determined.

Substituting the values of $a_{0}(X)$ and its derivative $a_{0}^{\prime}(X)$

From equation (57) and also the values of $a_{1}(X)$ and $h(X)$ obtained earlier into eqn. (56b), we obtain,

$g(X)=\left(\frac{1}{n}-1\right) A_{0}+\frac{A_{1}}{n} X+\left(1+\frac{1}{n}\right) A_{2} X^{2}$.

Substituting the values of $a_{0}(X), a_{1}(X)$ and $g(X)$ into eqn. (56c), we obtain

$\frac{\mathrm{n}\left(3 \beta^{2} \gamma+v-2 a \beta\right)}{\gamma} X^{2}+\frac{n\{(2 n+1) \lambda+2 v n\}}{\gamma(2 n+1)} X^{4}$ 


$$
=\left\{\begin{array}{c}
\left(\frac{1}{n}-1\right) A_{0}+\frac{A_{1}}{n} X \\
+\left(1+\frac{1}{n}\right) A_{2} X^{2}
\end{array}\right\}\left(A_{0}+A_{1} X+A_{2} X^{2}\right) .
$$

Equating coefficients of like powers of $X$ from both sides, we obtain,

$X^{0}:\left(\frac{1}{n}-1\right) A_{0}^{2}=0$.

$X:\left(\frac{2}{n}-1\right) A_{0} A_{1}=0$.

$X^{2}: \frac{2}{n} A_{0} A_{2}+\frac{A_{1}^{2}}{n}=\frac{n\left(3 \beta^{2} \gamma+v-2 a \beta\right)}{\gamma}$.

$X^{3}:\left(\frac{2}{n}+1\right) A_{1} A_{2}=0$.

$X^{4}:\left(1+\frac{1}{n}\right) A_{2}^{2}=\frac{n\{(2 n+1) \lambda+2 v n\}}{\gamma(2 n+1)}$.

Using Mathmatica, the solutions of the above set of equations are found to be

$A_{0}=0, A_{1}=0, A_{2}= \pm n \sqrt{\frac{\{(2 n+1) \lambda+2 v n\}}{\gamma(n+1)(2 n+1)}}$

$v=2 a \beta-3 \beta^{2} \gamma$.

Substituting these values of $A_{0}, A_{1}, A_{2}$ and hence of $a_{0}(X)$ and $a_{1}(X)$ into eqn. (54), we obtain,

$Y \pm n \sqrt{\frac{\{(2 n+1) \lambda+2 v n\}}{\gamma(n+1)(2 n+1)}} X^{2}=0$

Restoring the relations $X=U, Y=\frac{d X}{d \xi}=\frac{d U}{d \xi}$, we obtain,

$\frac{d U}{d \xi}=\mp n \sqrt{\frac{\{(2 n+1) \lambda+2 v n\}}{\gamma(n+1)(2 n+1)}} U^{2}$.

Further, remembering that $f(\xi)=U^{\frac{1}{n}}(\xi)$ and also

$q(x, t)=f(\xi) \exp [i\{\chi(\xi)-\omega t\}]$,

we obtain the solution in the form

$U= \pm \sqrt{\frac{\gamma(n+1)(2 n+1)}{n^{2}\{(2 n+1) \lambda+2 v n\}}} \xi^{-1}$

Or, $q(x, t)= \pm \exp \left[i\left\{\beta(x-v t)-\omega t+x_{0}\right\}\right]$

$\times\left[\sqrt{\frac{\gamma(n+1)(2 n+1)}{n^{2}\{(2 n+1) \lambda+2 v n\}}} \cdot \frac{1}{(x-v t)}\right]^{\frac{1}{n}}$

where $v=2 a \beta-3 \beta^{2}$.

We can find solutions for other values of $m$ also.

\section{Conclusion}

The first integral method has been successfully employed in solving the Kudryashov- Sinelshchikov equation and the Generalized Radhakrishnan-Kundu-Lakshmanan equation. It is seen that the performance of the method is quite well and we feel that the method is a powerful one in handling a wide variety of Nonlinear Evolution Equations available in many branches of Science and Engineering.

\section{References}

[1] Feng, Z.S., The first integral method to study the Burgers Korteweg-de Vries equation, J. Phys. A, 2002, 35 (2), 343 - 349. http://dx.doi.org/10.1088/0305-4470/35/2/312.

[2] Feng, Z. S.,On explicit exact solutions to the compound Burg ersKorteweg-de Vries equation, Phys. Lett. A. 2002, 293, 57 - 66. http://dx.doi.org/10.1016/S0375-9601(01)00825-8.

[3] Feng, Z. S., Travelling wave behavior for a generalized Fisher equation, Chaos, Soliton.Fract. 2008, 38, $481-488$. http://dx.doi.org/10.1016/j.chaos.2006.11.031.

[4] Feng, Z. S., Exact solution to an approximate sine- Gordon equation in $(\mathrm{n}+1)$ - dimensional space, Phys. Lett. A, 2002, 302, $64-$ 76. http://dx.doi.org/10.1016/S0375-9601(02)01114-3.

[5] Feng, Z. S. and Wang, X. H., The first integral method to the twodimensional Burgers- KdV equation, Phys. Lett. A 2002, 308, 173 - 178. http://dx.doi.org/10.1016/S0375-9601(03)00016-1.

[6] Feng, Z. S. and Knobel, R., Travelling waves to a BurgersKorteweg - de Vries equation with higher order nonlineari ties, J. Math. Anal. Appl. 2007, 328 (2), 1435 - 1450. http://dx.doi.org/10.1016/j.jmaa.2006.05.085.

[7] Raslan, R. K., The first integral method for solving some im portant nonlinear partial differential equations, Nonlinear Dynam. 2008, 53 (4), 281 - 286. http://dx.doi.org/10.1007/s11071-007-9262-x.

[8] Taghizadeh, N. Mirzazadeh, M. and Farahrooz, F., Exact solu tions of the nonlinear Schrodinger equation by the first inte gral method, J. Math. Anal. Appl. 2011, 374, 549 - 553. http://dx.doi.org/10.1016/j.jmaa.2010.08.050.

[9] Abbasbandy, S. and Shirzadi, A., The first integral method for modified Benjamin- Bona-Mahony equation, Commun. Nonlinear Sci. Numer.Simul. 2010, $15 \quad$ (7), 1759 - 1764. http://dx.doi.org/10.1016/j.cnsns.2009.08.003.

[10] Jafari, H. Sooraki, A. Telabi Y. and Biswas, A. , The first inte gral method and traveling wave solutions to Davey- Stewartson equation, Nonlinear Anal., Model. Control 2012, 17 (2) 182 -193.

[11] Taghizadeh, N., Mirzazadeh M. and Paghaleh, A. S., The First Integral Method to Nonlinear Partial differential Equations, Appl. Appl. Math. 2012, 7 (1), 117 - 132.

[12] Taghizadeh, N. Mirzazadeh, M. and Paghaleh, A. S., Exact solu tions for the nonlinear Schrodinger equation with power law nonlinearity, Math. Sci. Lett. 2012, 1 (1), 7 - 15 http://dx.doi.org/10.12785/msl/010102.

[13] El-Sabbagh, M. F. and El- Ganaini, S.I.,The First Integral Method and its Applications to Nonlinear Equations, Appl.Math. Sci., 2012, 6(78), 3893 - 3906.

[14] El- Dabe, N. T. M., Moussa, M. H. M., El- Shiekh R. M. and Hamdy, H. A., New Solutions for the Higher-Order Nonlinear Schrodinger Equation Using Integral Methods, American Jour na of Computational and Applied Mathemattics, 2012,2 (2), 25 - 28.

[15] El-Sabbagh , M. F. and El- Ganaini,, S.I., New Exact Solutions of Broer- Kaup (BK) and Whitham - Broer - Kaup (BWK) systems via the first integral method, Int. Journal of Math. Analysis 2012 61(46), $2287-2298$.

[16] Jafari, , H. Soltani, , R. Khalique ,C. M. and Baleanu, D., Exact solutions of two nonlinear partial differential equations by using the first integral method, Boundary Value Problems, 2013: 117. http://dx.doi.org/10.1186/1687-2770-2013-117.

[17] N. Taghizadeh, M. Mirzazadeh, Filiz Tascan, The first integra method applied to the Eckhaus equation, Appl. Math. Lett. 2012, 25,798 -802. http://dx.doi.org/10.1016/j.aml.2011.10.021.

[18] Taghizadeh, N., Mirzazadeh, M., Farahrooz F., Exact soliton solutions of the modified $\mathrm{KdV}-\mathrm{KP}$ equation and the Burgers- KP equation by using the first integral method, Appl. Math. Model. 2011 , $35(8)$, 3991-3997. http://dx.doi.org/10.1016/j.apm.2011.02.001.

[19] Kudryashov, N. A. and Sinelshchikov, D. I. Nonlinear waves in bubbly liquids with consideration for viscosity and heat transfer, Phys. Lett. A 2010, 374, 2011 - 2016. http://dx.doi.org/10.1016/j.physleta.2010.02.067.

[20] Kudryashov, N. A. and Sinelshchikov, D. I., Nonlinear evolu tion equations for describing waves in bubbly liquids with viscosity and heat transfer consideration, Appl. Math. Comput. 2010, 217, $414-$ 421. http://dx.doi.org/10.1016/j.amc.2010.05.033.

[21] Kudryashov, N. A. and Sinelshchikov, D. I., Nonlinear waves in liquids with gas bubbles with account of viscosity and heat transfer, Fluid Dynam. 2010, 45, $96 \quad-\quad 112$. http://dx.doi.org/10.1134/S0015462810010114.

[22] Mohammad Mirzazadeh, Mostafa Eslami, Exact solutions of the Kudryashov - Sinelshchikov equation and Nonlinear Tele graph 
equation via the first integral method, Nonlinear Anal. Model. 2012, $17(4), 481-488$.

[23] Sturdevant, b. lott d.a. and biswas, a., topological 1- soliton solution of the generalized radha krishnan kundu lakshmanan equation with nonlinear dispersion, modern physics let ters b, 2010, 24 (16), 1825 - 1831. http://dx.doi.org/10.1142/S0217984910024109.

[24] Anjan Biswas, (). 1 - Soliton solution of the generalized Radha krishnan, Kundu, Lakshmanan equation,Phys. Lett. A 2009, 373 (30), 2546 - 2548. http://dx.doi.org/10.1016/j.physleta.2009.05.010.

[25] Anjan Biswas, Kaiser R. Khan, Mohammad F. Mahmood \& Milivoj Belic,. Bright and Dark solitons in optical metamaterials, Optik 2014, $\quad 125(13), \quad 3299 \quad 3302$. http://dx.doi.org/10.1016/j.ijleo.2013.12.061. 